\title{
COVID-19 Outbreak: Key Considerations for Safety of Health Care Workers
}

\author{
Mohanjeet Kaur ${ }^{1}$ \\ ${ }^{1}$ Medical Director and Senior Medical Consultant, Shree Raghunath \\ Hospital, Ludhiana, Punjab, India
}

\begin{abstract}
Address for correspondence Mohanjeet Kaur, MD (Medicine), FICP, FIACM, Medical Director and Senior Medical Consultant, Shree Raghunath Hospital, Ludhiana, Punjab, India (e-mail: amarmohini@yahoo.in).
\end{abstract}

\begin{abstract}
Keywords

- COVID-19

- disinfection

- health care workers

- hydroxychloroquine prophylaxis

- personal protective equipment

- quarantine

- triage area

Since coronavirus disease 2019 (COVID-19) pandemic has unprecedented effects on both patients and health care workers (HCWs), we need to ponder upon various issues pertinent to HCWs, especially females. In this chapter, we intend to have an insight into challenges faced by HCWs regarding their risk profile, wearing personal protective equipment (PPE), and social, financial, and quarantine-related issues. We have tried to include data available so far regarding mortality of HCWs in fighting this epidemic. Also, health problems related to wearing PPE kits especially in female HCWs need to be addressed. Hydroxychloroquine prophylaxis for extended duration also needs well-defined guidelines.
\end{abstract}

\section{Introduction}

Coronavirus disease 2019 (COVID-19) pandemic has unprecedented effects on both patients and health care workers (HCWs). No disease has changed the way the health care is delivered to patients like this infection. Be it doctors, nursing staff, lab technicians, supporting staff of hospitals, and volunteers in health care system, the COVID-19 infection has taken a big toll on physical, social, psychological, economic, and professional aspects of life of HCWs.

Since data are being consolidated, the present information is gathered from the media, local resources, and studies done so far. We will be covering certain issues regarding working of HCWs in this writeup.

It is believed that 30 to $40 \%$ of HCWs are already exposed to COVID-19. According to the World Health Organization, till April 2020, over 22,000 HCWs worldwide had COVID-19 infection.

In a single-center case-series study done in Wuhan, 9,684 HCWs were enrolled. Data were collected from January 1, 2020, to February 2020. Overall, 110 HCWs were found to be positive for COVID-19, that is, an infection rate of $1.1 \%$. Out of the 110 infected HCWs, 70 (71.8\%) were women. One HCW (0.9\%) died in this study and 93 out of 110 HCWs (i.e., 84.5\%) had nonsevere disease.
Seventeen HCWs were posted in flu clinics. Also, $93 \mathrm{HCWs}$ out of $6,574(1.4 \%)$ were nonfirst-line workers. ${ }^{2}$

\section{Indian Data}

According to the available data, 106 doctors have lost their lives in India due to COVID infection so far. More than 50\% deaths were in the age group of below 60 years. ${ }^{3}$

The maximum death rate in doctors occurred in Maharashtra state (25\%) followed by Gujarat (11\%). - Fig. 1 shows the percentage of doctors' death in different states of India. ${ }^{3}$

According to the National COVID Registry of the Indian Medical Association Headquarters, Delhi, the state-wise distribution of infection rate among doctors in different states was registered. The registry reveals maximum number of infected doctors in Gujarat (520 out of 927) followed by Maharashtra (126 out of 927; - Fig. 2). ${ }^{3}$

\section{Other Issues}

Other issues that are involved include:

1. The HCWs have to create decontamination zones at home once back from daily duty.

2. Quarantine issues after duties in COVID hospital.
License terms

(ㄷ) (i) $\ominus \circledast$ 


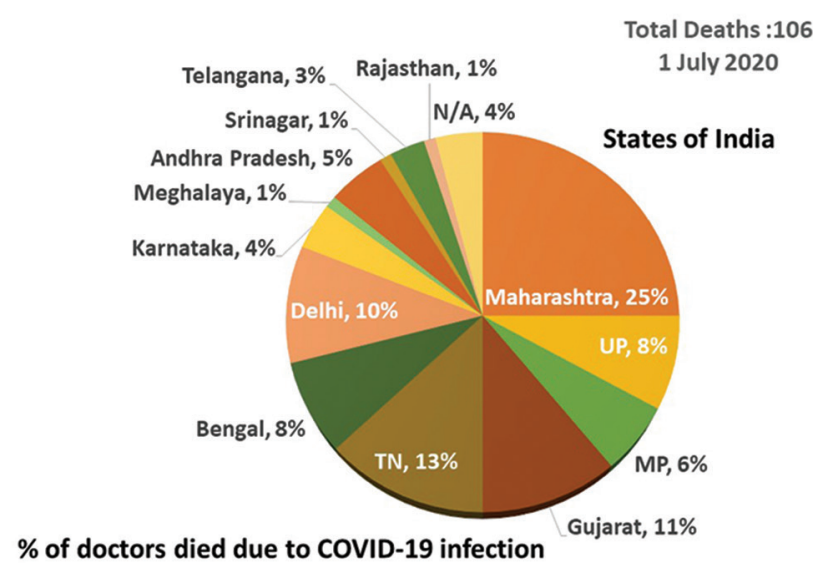

Fig. 1 Doctors' deaths in states of India, in percentage. COVID-19, coronavirus disease 2019.

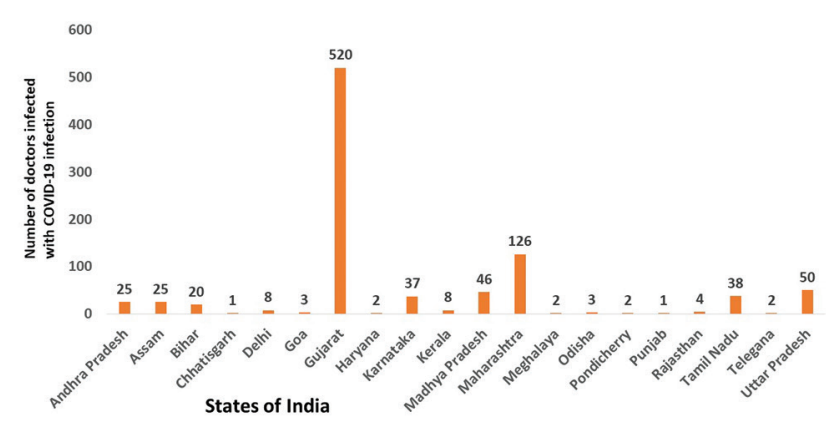

Fig. 2 Database of National Coronavirus Disease (COVID) Registry, Indian Medical Association (IMA) Headquarters, New Delhi (Doctors infected with COVID-19 in different states of India).

3. Facing social resistance, as there have been incidences of violence against HCWs by public.

4. Judicious availability of personal protective equipment (PPE) kits at workplace.

5. Procurement of certified N95 masks from the market.

6. Problems associated with wearing PPE kits especially during long duty hours. This is especially worth consideration in women HCWs, as $>70 \%$ are female HCWs in India facing issues like restrictions in using washrooms, menstrual health problems, and breast feeding.

7. Financial losses due to rotational duties of staff in health care setting.

8. Partial or complete shutting of hospitals or health care settings.

9. Medico-legal issues related to COVID-19 testing for HCWs themselves.

\section{Recommendations for HCWs in Health Care Settings}

1. All HCWs should undergo infection prevention and control program. ${ }^{4}$

2. Regular thermal screening for HCWs should be done.

3. The cleaning and disinfection of environmental surfaces should be done as per standard operating procedure

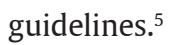

4. HCWs should follow hand hygiene, social distancing, and cough etiquettes themselves also.

5. Triage area/flu corner should be made in all health care setting.

6. All patients should be screened at the entrance of the hospitals using noncontact temperature and oxygen saturation via pulse oximeter. The area and pulse oximeter should be cleaned after each use.

7. Soap and water should be made available adequately to avoid unnecessary use of sanitizers.

8. Regular disinfection of phone, pages, badge, eye covers, stethoscopes, etc. should be done.

9. After coming in contact with a COVID patient, HCWs who have not used PPE (high risk) must be strictly quarantined (home/institution) for 14 days. Testing for COVID-19 should be done after 5 days of exposure.

10. HCWs with low risk need not be quarantined. However, they should self-monitor their symptoms.

11. HCWs detected positive need to be isolated appropriately.

12. The hospital staff should wear PPE depending upon their risk profile and area of work.

13. Staff should do duty in rotation to decrease exposure time.

14. Aerosolized procedures should be performed with proper precaution.

15. Isolation facility and COVID ward should be set up as per government guidelines. ${ }^{6}$

16. Setting up of COVID dedicated operation theaters and dental clinics should be done. ${ }^{7,8}$

17. Taking care of patients who died due to COVID-19 infection. There have been incidences where last rituals are also performed by HCWs only.

\section{Risk Profile of HCWs and Use of PPE}

For safety of HCWs, protective equipment are recommended to minimize the exposure to a biological agent. The Government of India has issued recommendations regarding rational use of PPE for HCWs based on duty areas and risk profile. ${ }^{9,10}$ These health care settings are divided into two parts (-Table 1):

1. COVID areas.

2. Non-COVID settings with COVID facilities.

The guidelines for donning and doffing are given in - Table 2.

Health problems associated with masks/PPE kits:

1. Hypersensitivity reactions (e.g., glove powder).

2. Fatigue, dizziness, headache, and feeling of suffocation due to hypoxygenemia after wearing N95 mask for a long duration or irritability and subjective perception of discomfort. ${ }^{11}$

3. Menstrual health problems and restriction of using washrooms especially in female HCWs.

\section{Risk Score for HCWs for COVID-Associated Mortality}

The following factors have been associated with increased mortality rate in COVID-19 infection. ${ }^{12}$ 
Table 1 Levels of PPE formulated for out- and inpatient departments and supportive areas

\begin{tabular}{|l|l|l|}
\hline Services & COVID settings & $\begin{array}{l}\text { Non-COVID settings with COVID } \\
\text { facilities }\end{array}$ \\
\hline OPD area (including triage area and reception) & N95 mask + gloves & Triple layer mask + gloves \\
\hline IPD (ICU + critical care) & Complete PPE & N95 mask + gloves + face shield \\
\hline Emergency & Complete PPE & $\begin{array}{l}\text { - Routine cases: triple mask + gloves } \\
\text { R Respiratory illness: complete PPE }\end{array}$ \\
\hline Supportive areas & \multicolumn{2}{|l}{} \\
\hline Lab & Complete PPE & Triple layer mask + gloves \\
\hline Sanitary staff & N95 mask + gloves & Triple layer mask + gloves \\
\hline Drivers & & \multicolumn{2}{|l|}{} \\
\hline - Patients with respiratory distress & Full PPE & Triple layer mask + gloves \\
\hline - Patients without respiratory distress & N95 mask + gloves &
\end{tabular}

Abbreviations: COVID, coronavirus disease; ICU, intensive care unit; OPD, outpatient department; IPD, inpatient department; PPE, personal protective equipment.

Table 2 Steps for donning and doffing

\begin{tabular}{|l|l|}
\hline Donning sequence & Doffing sequence \\
\hline Hand wash & Outer gloves \\
\hline Cap & Hood \\
\hline Shoe cover & Coverall/gown \\
\hline Hand rub & Shoe cover \\
\hline Inner glove & Hand rub (gloved hand) \\
\hline Coverall/gown & Goggles \\
\hline Mask (surgical or N95) & Mask (surgical or N95) \\
\hline Goggles & Cap \\
\hline Hood & Inner glove \\
\hline Outer gloves & Hand wash \\
\hline
\end{tabular}

Note: The areas for donning and doffing should be separate. Appropriate disposal of PPE kit should be done. All external wearing such as watches, jewelry, ear rings, bangles, pen, identity card should be removed before donning. Mask should be removed only outside patient room.

1. Age more than 65 years

2. Male gender

3. Hypertension

4. Diabetes mellitus

5. Cardiovascular disease

6. Chronic obstructive pulmonary disease

7. Malignancies

\section{HCQ Prophylaxis in HCWs}

On May 22, 2020, the Indian Council of Medical Research (ICMR) released guidelines for hydroxychloroquine (HCQ) prophylaxis in India. It was submitted to pharmacovigilance program of India. ${ }^{13}$

HCQ prophylaxis should be given to:

- HCWs in COVID and non-COVID areas.

- Other people, for example, policemen who are working for control of COVID infection and staff involved in door-to-door surveillance and screening. Exclusion Criteria

- HCW with cardiomyopathy or cardiac rhythm disorder.

- Retinopathy.

- People sensitive to HCQ.

- Persons with G6PD deficiency.

Note that HCQ is not recommended for prophylaxis in pregnancy and lactation.

\section{Dose of HCQ for Prophylaxis in HCWs}

The recommended dose is $400 \mathrm{mg} \mathrm{BD} \times 1$ day, then $400 \mathrm{mg}$ once a week for 7 weeks. However, as per ICMR guidelines, its use after 8 weeks is also recommended.

\section{ECG Monitoring}

- Baseline electrocardiogram (ECG) with QT interval estimate calculation may be done before starting HCQ prophylaxis.

- ECG should be repeated if the person develops cardiovascular symptoms, for example, palpitations, chest pain, or syncope.

- ECG should be done in persons where HCQ is extended beyond 8 weeks.

- ECG should be done anytime during the duration of prophylaxis.

\section{Special Precautions}

- HCQ should be given by a registered medical practitioner.

- Along with HCQ prophylaxis, adequate protection with PPE kits should be done.

- The HCW should watch for any adverse effect of prophylaxis.

- Appropriate treatment should be taken if HCWs develop fever, cough, etc. while on HCQ prophylaxis.

- HCQ prophylaxis does not substitute preventive measures like hand hygiene and social distancing.

- Even if they are on prophylactic treatment, the HCWs, after coming in contact with a proven positive case, should opt for quarantine. 


\section{Conclusion}

The COVID-19 pandemic has changed the overall scenario of the health care system across the globe. The unavailability of vaccine so far needs more gearing up of the health care system. So, we, as HCWs, have a long way to go, keeping the balance between delivering our duties diligently and being careful about ourselves and our staff who are helping us in fighting this pandemic.

\section{Conflict of Interest}

None.

\section{References}

1 WHO says over 22,000 healthcare workers across 52 countries infected by COVID-19. Available at: https://health. economictimes.indiatimes.com/news/industry/who-says-over22000-healthcare-workers-across-52-countries-infectedby-covid-19/75107238. Accessed Aug 19, 2020

2 Lai X, Wang M, Qin C, et al. Coronavirus disease 2019 (COVID-2019) infection among health care workers and implications for prevention measures in a tertiary hospital in Wuhan, China. JAMA Netw Open 2020;3(5):e209666

3 Dr Rajeev Jayadevan. A hundred lives lost: doctor deaths in India during the times of COVID-19. Preprints 2020, 2020070346 (doi:10.20944/preprints202007.0346.v1).Availableat:(https:// www.preprints.org/manuscript/202007.0346/v1). Accessed Aug 19, 2020

4 Advisory for managing health care workers working in COVID and non-COVID areas of the hospital. Available at: https://www. mohfw.gov.in/pdf/updatedAdvisoryformanagingHealthcareworkersworkinginCOVIDandNonCOVIDareasofthehospital.pdf. Accessed Aug 19, 2020

5 Cleaning and disinfection of environmental surfaces in the context of COVID-19. Available at: https://www.who.int/ publications/i/item/cleaning-and-disinfection-of-environmentalsurfaces-inthe-context-of-covid-19. Accessed Aug 19, 2020
6 National Centre for Disease control, Ministry of Health and Family Welfare. Covid-19 outbreak guidelines for setting up isolation facility/ward. Available at: https://ncdc.gov.in/ WriteReadData/1892s/42417646181584529159.pdf. Accessed Aug 19, 2020

7 Guidelines for dental professionals in COVID-19 pandemic situation.Availableathttps://www.mohfw.gov.in/pdf/DentalAdvisoryF. pdf. Accessed Aug 19, 2020

8 Lillemoe KD. Surgical perspectives: an introduction. Ann Surg 2014;259(6):1037

9 Ministry of Health and Family Welfare, Directorate General of Health Services. Novel coronavirus disease 2019 (COVID-19): guidelines on rational use of personal protective equipment. Available at: https://www.mohfw.gov.in/pdf/ GuidelinesonrationaluseofPersonalProtectiveEquipment.pdf. Accessed Aug 19, 2020

10 Ministry of Health and Family Welfare, Directorate General of Health Services. Novel coronavirus disease 2019 (COVID-19): additional guidelines on rational use of personal protective equipment (setting approach for health functionaries working in non-COVID areas). Available at: https://www.mohfw. gov.in/pdf/AdditionalguidelinesonrationaluseofPersonal ProtectiveEquipment settingapproachfor Healthfunctionaries workinginnonCOVIDareas.pdf. Accessed August 1, 2020

11 Li Y, Tokura H, Guo YP, et al. Effects of wearing N95 and surgical facemasks on heart rate, thermal stress and subjective sensations. Int Arch Occup Environ Health 2005;78(6):501-509

12 Parohan M, Yaghoubi S, Seraji A, Javanbakht MH, Sarraf P, Djalali M. Risk factors for mortality in patients with coronavirus disease 2019 (COVID-19): a systematic review and meta-analysis of observational studies. Available at: https:// www.medrxiv.org/content/10.1101/2020.04.09.20056291v2. Accessed Aug 19, 2020

13 Revised advisory on the use of hydroxychloroquine (HCQ) as prophylaxis for COVID-19 infection (in supersession of previous advisory dated 23rd March, 2020). Available at: https://www.mohfw.gov.in/pdf/Revisedadvisoryontheuse ofhydroxychloroquineasprophylaxisforSARSCOVID19infection.pdf. Accessed Aug 19, 2020 